\title{
Two-Dimensional DNA Electrophoresis Identifies Novel CpG Islands Frequently Coamplified With MYCN in Neuroblastoma
}

\author{
K. Wimmer, ${ }^{1,2 *}$ X.X. Zhu, ${ }^{1}$ B.J. Lamb, ${ }^{1}$ R. Kuick, ${ }^{1}$ P. Ambros, ${ }^{3}$ H. Kovar, ${ }^{3}$ \\ D. Thoraval, ${ }^{1+}$ A. Elkahloun, ${ }^{4}$ P. Meltzer, ${ }^{4}$ and S.M. Hanash ${ }^{1}$
}

\begin{abstract}
Background. Amplification of the oncogene MYCN in neuroblastoma has been found to correlate with aggressive tumour growth and is used as a predictor of clinical outcome. The MYCN amplicon is known to involve coamplification of extensive DNA regions. Therefore it is possible that other genes are coamplified in this amplicon and that they may play a role in the poor outcome of MYCN amplified tumours. Procedure. We have implemented an approach for the two-dimensional separation of human genomic restriction fragments to detect and isolate as yet unknown amplified sequences in the MYCN amplicon in neuroblastoma. Using this approach we have recently cloned a novel
\end{abstract}

gene referred to as NAG that is frequently coamplified with MYCN in neuroblastoma. $\boldsymbol{R e}$ sults and Conclusions. We report here the identification and cloning of two ad-ditional CpG islands that are amplified in neuroblastoma. One contains a sequence that is identical to the first intron of $D D X 1$. The other represents a novel CpG island that is associated with an as yet unidentified gene. We show that the novel CpG island is located in close proximity to the MYCN locus on chromosome 2 and is as frequently coamplified with $M Y C N$ in neuroblastoma as NAG and DDX1. Med. Pediatr. Oncol. 36:75-79, 2001. @ 2001 Wiley-Liss, Inc.

Key words: gene amplification; neuroblastoma; MYCN amplicon

\section{INTRODUCTION}

Genomic amplification is a common mechanism leading to deregulated gene expression in human cancers. In neuroblastoma, MYCN amplification has been found to correlate with aggressive tumour growth and can serve as a predictor of clinical outcome $[1,2]$. There is evidence that amplification rather than up-regulation of basal expression is the main mechanism for the loss of autoregulation of $M Y C N$ [3] and its overexpression [4,5]. However, it is not clear how MYCN amplification results in poor outcome [6-8]. It is possible that coamplification of other DNA sequences plays a role in the poor outcome of MYCN amplified tumours.

Although the $M Y C N$ amplicon is known usually to involve coamplification of extensive regions of DNA, only the DEAD-box gene $D D X 1$, to date, has been identified as being frequently coamplified with $M Y C N$ in neuroblastoma $[9,10]$. We have implemented an approach for the two-dimensional (2-D) separation of human genomic restriction fragments to detect novel genomic alterations in neuroblastoma cell lines and primary tumours [11,12]. The 2-D approach allows the identification and isolation of as yet unknown amplified sequences. The use of the enzyme NotI to digest DNA and the tagging of the fragments at the NotI ends allow preferential visualization and quantitative analysis of fragments containing $\mathrm{CpG}$ islands. Using this approach we have recently cloned a novel gene referred to as $N A G$, that is frequently coamplified with $M Y C N$ in neuroblas- toma [13]. We report here the identification and cloning of two additional $\mathrm{CpG}$ islands that are amplified in neuroblastoma. One is identified as containing the first intron of $D D X 1$, and the other represents a novel $\mathrm{CpG}$ island that is associated with an as yet unidentified gene. We show that the novel CpG island is frequently coamplified with $M Y C N$.

\section{MATERIALS AND METHODS Cell Culture}

Human neuroblastoma cell lines SKNSH [14], IMR32 [15], KCNR [16], and NGP127 [17] have been characterized earlier. All STA-NB-3, -4, -8, -9, and -11 cell lines [18] have been established at the Children's Cancer Research Institute (Vienna, Austria) and showed the same karyotypic pattern as the tumors from which they were derived.

\footnotetext{
${ }^{1}$ Department of Pediatrics, University of Michigan, Ann Arbor, Michigan

${ }^{2}$ Department of Medical Biology, University of Vienna, Vienna, Austria

${ }^{3}$ Children's Cancer Research Institute, Vienna, Austria

${ }^{4}$ National Human Genome Institute, Bethesda, Maryland

${ }^{\dagger}$ Current address: Laboratoire de Biologie Moleculaire et de Sequencage (LBMS), IBGC du CNRS, Universite de Bordeaux II (BP 64), 146 rue Leo Saignat- 33076, Bordeaux Cedex, France.

*Correspondence to: K. Wimmer, Department of Medical Biology, University of Vienna, Wäringerstrasse 10, A-1090 Vienna, Austria.

E-mail: katharina.wimmer@unive.ac.at
} 
Wimmer et al.
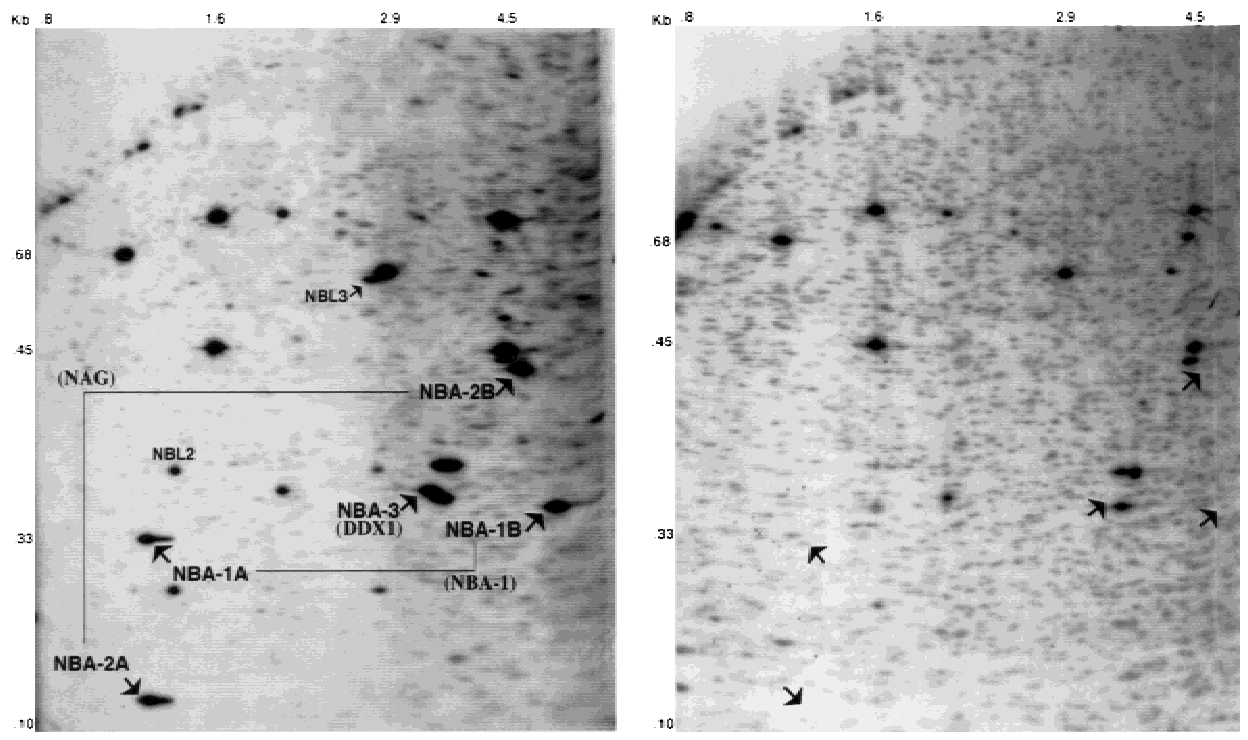

Fig. 1. Images of 2-D gels for neuroblastoma cell line STA-NB11 (left) and peripheral blood lymphocytes from the patient from whose tumor the cell line was derived (right). Large arrows point to spots with increased intensity owing to the fragments being amplified. Thin lines join pairs of spots whose fragments share the same NotI restriction site. The names for genes are shown in parentheses. Small arrows point to fragments from tandemly repeated DNA that is often demethylated in neuroblastomas $[11,23]$.

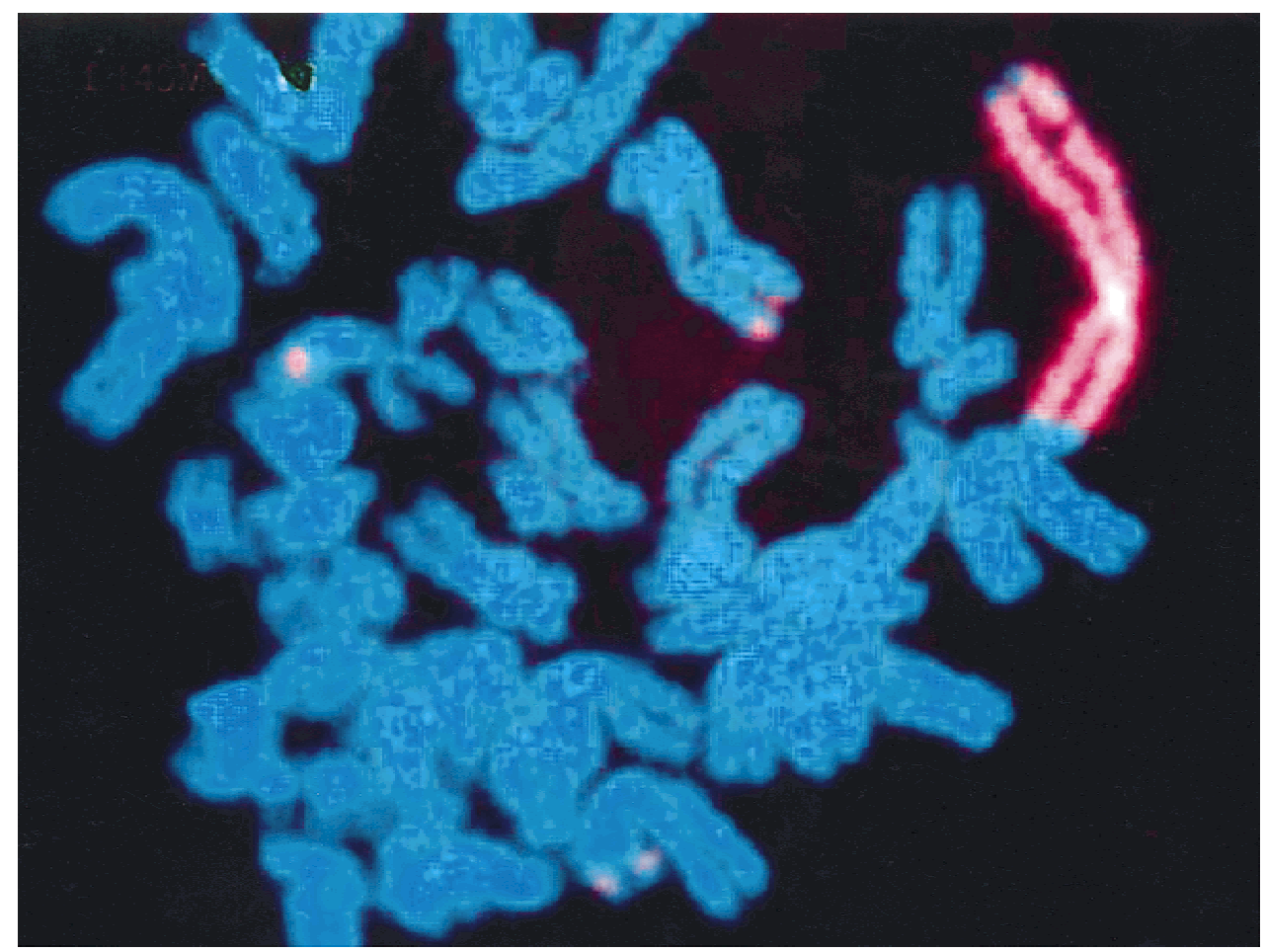

Fig. 2. Fluorescence in situ hybridisation of the $\mathrm{CpG}$ island NBA-1 on metaphase chromosomes of neuroblastoma cell line NGP127. The BAC clone containing $\mathrm{CpG}$ island $N B A-1$ was labeled with biotin and visualized with TRITC-labeled antibodies. Chromosomes were counterstained with DAPI.

\section{2-D Gel Analysis}

A detailed description of the experimental conditions can be found in [11]. Briefly, genomic DNA was digested with NotI and EcoRV restriction enzymes, and the NotI derived $5^{\prime}$ protruding ends were ${ }^{32} \mathrm{P}$-labeled. After first-dimensional separation of the fragments in agarose disc gels, a second digestion was done in situ with DpnII. The resulting fragments were separated perpendicularly in a polyacrylamide gel. 2-D gels were dried and exposed to PhosphoImager plates (Molecular Dynamics, Sunnyvale, CA). Digital images were obtained after scanning of the PhosphoImager plates.

\section{Cloning of the Amplified DNA Fragments}

DNA fragments were cloned from preparative gels as previously described [11]. Briefly, both radiolabeled and 


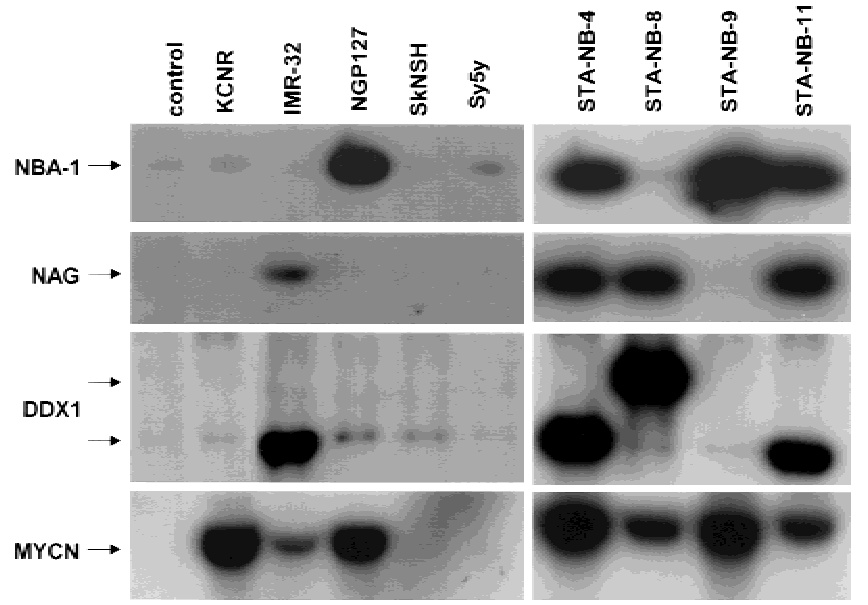

Fig. 3. Southern blot detection of $M Y C N, D D X 1, N A G$, and $N B A-1$ amplification in neuroblastoma cell lines. Genomic DNA of nine cell lines and of peripheral blood lymphocytes of a healthy donor (control) was digested with NotI and EcoRV, the enzymes for the firstdimensional separation in the 2-D analysis. The filter was hybridized sequentially with a $0.8 \mathrm{~kb}$ fragment of the $M Y C N$ probe $\mathrm{pNb}-1$ and with the cloned $N o t \mathrm{I} / D p n I \mathrm{II}$ fragment $N B A-3, N B A-2 A$, and $N B A-1 A$ as probe for $D D X 1, N A G$, and the $\mathrm{CpG}$ island $N B A-1$, respectively. The $D D X 1$ probe detects two different NotI/EcoRV fragments of $3.4 \mathrm{~kb}$ and $6.0 \mathrm{~kb}$, which may represent a polymorphism for one of the restriction sites.

nonradiolabeled genomic digests of cell line STA-NB-11 were loaded onto first-dimension agarose gels. After the second-dimension separation in polyacrylamide, the gels were exposed to X-ray film. The NotI/DpnII fragments corresponding to the DNA spots of interest were recovered and ligated in a NotI/BamHI digested $\mathrm{pBC}$-vector (ClonTech, Palo Alto, CA) in the presence of DNA ligase at $16^{\circ} \mathrm{C}$ for $40 \mathrm{hr}$. The transformation was performed by electroporation with the Epicurian Coli XL1-Blue MRF electroporation-competent cells (Stratagene, La Jolla, CA).

\section{RESULTS AND DISCUSSION \\ 2-D DNA Electrophoresis Identifies CpG Islands Frequently Coamplified With MYCN in Neuroblastoma}

We have undertaken an initial 2-D DNA electrophoresis analysis for six neuroblastoma cell lines. The 2-D patterns obtained were compared to a series of more than 200 patterns of a variety of normal tissues and other malignant and nonmalignant cell lines in order to identify multicopy fragments that are derived from amplified DNA in the neuroblastoma cell lines. Five Not I/DpnII fragments designated $N B A-1 A,-1 B, 2 A,-2 B$, and -3 (Fig. 1 ) were present in multiple copies only in neuroblastoma cell lines that contain an amplified $M Y C N$ gene as determined by Southern analysis. However, none of the five fragments was derived from the $M Y C N$ gene; the latter

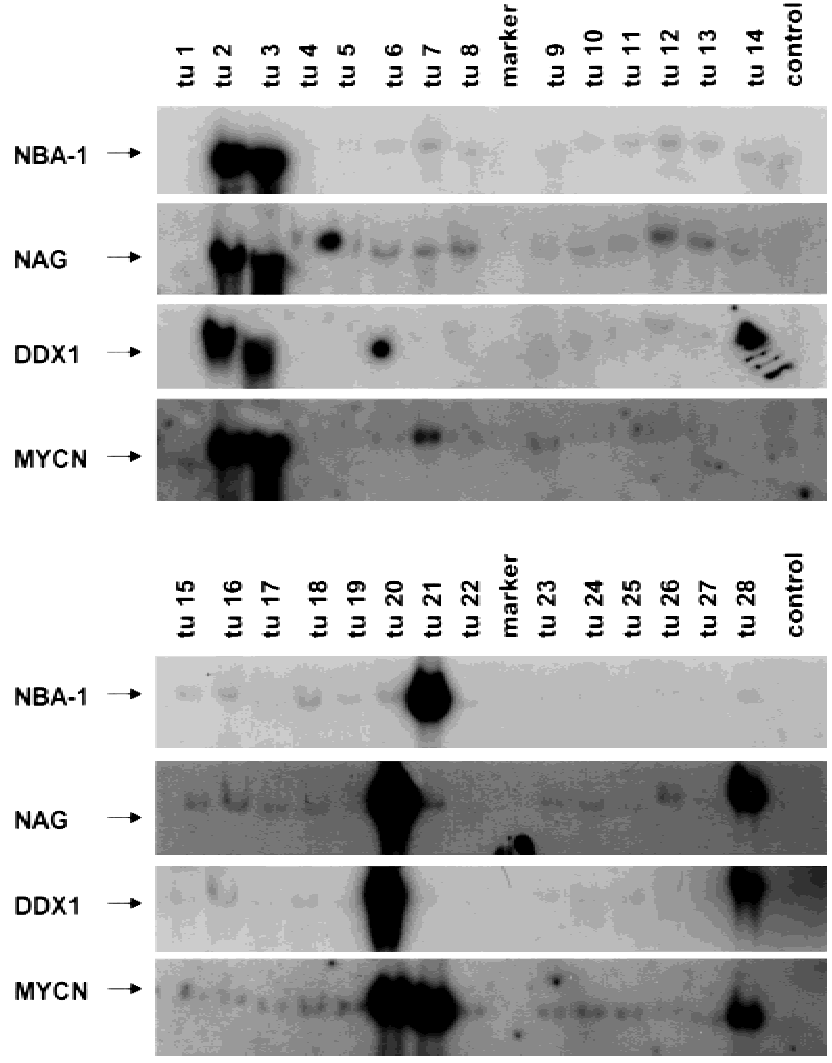

Fig. 4. Southern blot detection of $M Y C N, D D X 1, N A G$, and $N B A-1$ amplification in neuroblastoma tumours. Tumour DNA and DNA of peripheral blood lymphocytes of a healthy donor (control) were digested with BamHI. The filters were hybridized sequentially with probes as described for Figure 3.

yields, with the enzyme combination utilized, fragments of $>10 \mathrm{~kb}$ that are outside the first-dimension separation range.

Two fragments designated $N B A-2 A$ and $-2 B$ were recently cloned and shown to be part of a novel gene, which we have cloned and referred to as $N A G$ [13]. NAG was shown to be frequently coamplified with $M Y C N$. In this study we cloned the three other multicopy Not/DpnII fragments $N B A-1 A,-1 B$, and -3 from preparative 2-D gels as described in Materials and Methods. The cloned fragments were sequenced, and the novel sequences were compared to a known sequences from the EMBL database. Starting at the NotI site a stretch of $181 \mathrm{bp}$ of the 290 bp fragment $N B A-3$ was identical with a $\mathrm{CpG}$ island in the database. This sequence also matches the sequence for the first intron of $D D X 1$ (R. Godbout, personal communication). Fragments $N B A-1 A$ and $-1 B$ showed no significant homology with any of the known sequences in the database. Using the $253 \mathrm{bp}$ fragment $N B A-1 A$ as a probe, we isolated a BAC clone (BAC14876). A $6.3 \mathrm{~kb}$ PstI fragment of this BAC clone that encompassed NBA$1 A$ was subcloned. Partial sequence analysis of the subclone revealed that the $287 \mathrm{bp}$ fragment NBA-1B is en- 
compassed in the BAC subclone and that fragments $N B A-1 A$ and $-1 B$ contained (one at its $5^{\prime}$ end and the other at its $3^{\prime}$ end) the same $\operatorname{NotI}$ restriction site and will thus be referred to together as NBA-1. Approximately $1,023 \mathrm{bp}$ of the BAC subclone sequence surrounding the NotI site revealed the characteristics of a $\mathrm{CpG}$ island with a $\mathrm{C}$ and $\mathrm{G}$ content of $65 \%$ of the nucleotides and a $\mathrm{CpG} / \mathrm{GpC}$ ratio of 0.61 . Thus it is likely that NBA-1 represents a novel $\mathrm{CpG}$ island that is associated with an as yet unidentified gene. Using the BAC clone as probe, we performed fluorescence in situ hybridization (FISH) on metaphases of neuroblastoma cell line NGP127 (Fig. 2 ), which exhibits amplification of the NBA-1 fragment. Bright signals are observed at the chromosome bands 2p23-24 confirming the localization of NBA-1 in close vicinity to the locus of the $M Y C N$ gene. Furthermore, hybridization signals in the HSR integrated at chromosome band $4 \mathrm{p} 16$ confirmed the coamplification of NBA-1 with MYCN in the HSR of this cell line [19].

\section{Comparison of the Frequency of Coamplification of DDX1, NAG, and fragment NBA-1 With MYCN}

Because NBA- 1 was found to be amplified in three of the four $M Y C N$-amplified neuroblastoma cell lines in our initial analysis, it was likely that this $\mathrm{CpG}$ island is frequently encompassed in the $M Y C N$ amplicon in neuroblastoma as $D D X 1$ and $N A G$. To compare the frequency of coamplification of $D D X 1, N A G$, and the newly cloned CpG island with $M Y C N$, we utilized for Southern analysis the cloned NotI/DpnII fragments NBA-3 and NBA-2A, and $N B A-1 A$ as probes for $D D X 1, N A G$, and the newly cloned $\mathrm{CpG}$ island, respectively (Figs. 3, 4). A total of 10 neuroblastoma cell lines and a total of 65 neuroblastoma tumor samples were analyzed. Amplification of either of the three fragments was found only in neuroblastomas that were also amplified for $M Y C N$. In two of the eight $M Y C N$-amplified cell lines (STA-NB-4 and STA-NB-11) and in five of thirteen $M Y C N$-amplified tumors, all three fragments were amplified. In three cell lines (IMR-32, STA-NB-3, STA-NB-8) and in four tumours, both DDX1 and $N A G$ were coamplified with $M Y C N$. Cell lines NGP127 and STA-NB-9 and the remaining four $M Y C N$ amplified tumors showed coamplification of only the $N B A-3$ fragment with $M Y C N$. Thus we found that $D D X 1$ and $N A G$ were coamplfied in five of eight $(62 \%)$ cell lines and in nine of thirteen $(70 \%)$ of the tumors, and there were no cases in which one was amplified without the other. The NBA-1 CpG island was coamplified with $M Y C N$ in four of eight (50\%) of the cell lines and nine of thirteen $(70 \%)$ of the tumours. Cell line KCNR but none of the tumors showed amplification of only the $M Y C N$ gene.

The MYCN amplicon contains two NotI sites located less then $50 \mathrm{~kb}$ from each other, within a $500 \mathrm{~kb}$ region at the $5^{\prime}$ end of MYCN [20]. It is likely that the CpG islands of $D D X 1$ and $N A G$, which were always coamplified in our series of neuroblastomas, contain the two NotI restriction sites. The $\mathrm{CpG}$ island encompassed by fragment $N B A-1$ shows a partly overlapping but distinct pattern of coamplification compared to $D D X 1$ and $N A G$. Thus it may be presumed that $N B A-1$ is located at the $3^{\prime}$ end of MYCN. According to Akiyama et al. [20], the closest NotI site $3^{\prime}$ of $M Y C N$ has been found at a distance of $680 \mathrm{~kb}$.

Amplification of $M Y C N$ is a well-established prognostic factor in neuroblastoma, and ovenexpression of $M Y C N$ can contribute to cell transformation [21] and targeted expression of $M Y C N$ results in neuroblastoma in transgenic mice [22]. However, given that the MYCN amplicon contains additional genes including $D D X 1$, $N A G$, or yet a third gene associated with the $\mathrm{CpG}$ island $N B A-1$, the heterogeneity in the MYCN amplicon may influence the biology of neuroblastoma.

\section{REFERENCES}

1. Schwab M, Amler LC. Amplification of cellular oncogenes: a predictor of clinical outcome in human cancer. Genes Chrom Cancer 1990;1:181-193.

2. Schwab M, Corvi R, Amler LC. MYCN oncogene amplification: a consequence of genomic instability in human neuroblastoma. Neuroscientist 1995;1:277-285.

3. Sivak LE, Tai KF, Smith RS, Dillon PA, Brodeur GM, Carroll WL. Autoregulation of the human N-myc oncogene is disrupted in amplified but not single-copy neuroblastoma cell lines. Oncogene 1997; $15: 1937-1946$.

4. De Bernardi B, Conte M, Mancini A, et al. Localized resectable neuroblastoma: results of the second study of the Italian Cooperative Group for Neuroblastoma. J Clin Oncol 1995;13:884-893.

5. Lutz W, Schwab M. In vivo regulation of single copy and amplified N-myc in human neuroblastoma cells. Oncogene 1997;15: 303-315.

6. Hiyama E, Hiyama K, Yokoyama T, Ishii T. Immunohistochemical analysis of $\mathrm{N}-m y c$ protein expression in neuroblastoma: correlation with prognosis of patients. J Pediatr Surg 1991;26:838843.

7. Nisen PD, Waber PG, Rich MA, et al. N-myc oncogene RNA expression in neuroblastoma. JNCI 1988;80:1633-1637.

8. Slavc I, Ellenbogen R, Jung W-H, et al. myc gene amplification and expression in primary human neuroblastoma. Cancer Res 1990;50:1459-1463.

9. George RE, Kenyon RM, McGuckin AG, Malcolm AJ, Pearson ADJ, Lunec J. Investigation of co-amplification of the candidate genes ornithine decarboxylase, ribonucleotide reductase, syndecan-1 and a DEAD box gene, DDXI, with N-myc in neuroblastoma. Oncogene 1996;12:1583-1587.

10. Squire JR, Thorner PS, Weitzman S, et al. Co-amplification of $M Y C N$ and a DEAD box gene $(D D X I)$ in primary neuroblastoma. Oncogene 1995;10:1417-1422.

11. Thoraval D, Asakawa J, Kodaira M, et al. A methylated human 9 $\mathrm{kb}$ repetitive sequence on acrocentric chromosomes is homologous to a subtelomeric repeat in chimpanzees. Proc Natl Acad Sci USA 1996;93:4442-4447.

12. Wimmer K, Thoraval D, Kuick R, Lamb BJ, Hanash SM. Identification of amplifications, deletions, and methylation changes in cancer by means of two-dimensional analysis of genomic digests: 
application to neuroblastoma. Biochem Soc Trans 1997;25:262267.

13. Wimmer K, Zhu X-X, Lamb BJ, et al. Co-amplification of a novel gene, $N A G$, with the $\mathrm{N}-m y c$ gene in neuroblastoma. Oncogene 1999;18:223-238.

14. Biedler JL, Helson L, Spengler BA. Morphology and growth, tumorigenicity, and cytogenetics of human neuroblastoma cells in continuous culture. Cancer Res 1973;33:2643-2652.

15. Tumilowicz JJ, Nichols WW, Cholon JJ, Greene AE. Definition of a continuous human cell line derived from neuroblastoma. Cancer Res 1970;30:2110-2118.

16. Reynolds CP, Biedler JL, Spengler BA, et al. Characterization of human neuroblastoma cell lines established before and after therapy. JNCI 1986;76:375-387.

17. Amler LC, Corvi R, Praml C, Savelyeva L, Le Paslier D, Schwab M. A reciprocal translocation $(1 ; 15)(36.2 ; \mathrm{q} 24)$ in a neuroblastoma cell line is accompanied by DNA duplication and may signal the site of a putative tumor suppressor-gene. Oncogene 1995;10: 1095-1101.

18. Ambros IM, Rumpler S, Luegmayr A, et al. Neuroblastoma cells can actively eliminate supernumerary $M Y C N$ gene copies by micronucleus formation - sign of tumour cell revertance? Eur J Cancer 1997;33:2043-2049.

19. Van Roy N, Forus A, Myklebost O, Cheng NC, Versteeg R, Speleman F. Identification of two distinct chromosome 12-derived amplification units in neuroblastoma cell line NGP. Cancer Genet Cytogenet 1995;82:151-154.

20. Akiyama K, Kanda N, Yamada M, Tadokoro K, Matsunaga T, Nishi Y. Megabase-scale analysis of the origin of N-myc amplicons in human neuroblastomas. Nuclear Acids Res 1994;25:187193.

21. Schwab M, Varmus HE, Bishop JM. Human N-myc gene contributes to neoplastic transformation of mammalian cells in culture. Nature 1985;316:160-162.

22. Weiss WA, Aldape K, Mohapatra G, Feuerstein BG, Bishop JM. Targeted expression of MYCN causes neuroblastoma in transgenic mice. EMBO J 1997;16:2985-2995.

23. Thoraval D, Asakawa J, Wimmer K, et al. Demethylation of repetitive DNA sequences in neuroblastoma. Genes Chrom Cancer 1996;17:234-244. 